\title{
Holographic Random Access Memory (HRAM)
}

\author{
ERNEST CHUANG, WENHAI LIU, JEAN-JACQUES P. DROLET, ASSOCIATE MEMBER, IEEE, \\ AND DEMETRI PSALTIS, SENIOR MEMBER, IEEE
}

\section{Invited Paper}

\begin{abstract}
We examine the present state of holographic random access memory (HRAM) systems and address the primary challenges that face this technology, specifically size, speed, and cost. We show that a fast HRAM system can be implemented with a compact architecture by incorporating conjugate readout, a smart-pixel array, and a linear array of laser diodes. Preliminary experimental results support the feasibility of this architecture. Our analysis shows that in order for the HRAM to become competitive, the principal tasks will be to reduce spatial light modulator (SLM) and detector pixel sizes to $1 \mu \mathrm{m}$, increase the output power of compact visible-wavelength lasers to several hundred milliwatts, and develop ways to raise the sensitivity of holographic media to the order of $1 \mathrm{~cm} / \mathrm{J}$.
\end{abstract}

Keywords-Holographic memories, laser array, phase conjugation, photorefractive effect, random access memories.

\section{INTRODUCTION}

Holographic memory has received attention in recent years as a technology that can provide very large storage density and high speed [1]-[5]. Information is recorded in the holographic medium through the interference of two coherent beams of light. We refer to the informationcarrying beam as the signal beam and the interfering beam as the reference beam. The resulting interference pattern causes an index grating (the hologram) to be written in the material. When the hologram is subsequently illuminated with one of the original reference beams, light is diffracted from the grating in such a way that the signal beam is reproduced. Many holograms can be multiplexed within the same volume of the material by angle [6], [7], fractal [8], wavelength [9], [10], phase code [11]-[13], peristrophic [14], and shift [15], [16] multiplexings.

Manuscript received October 9, 1998; revised April 8, 1999. This work was supported in part by Jet Propulsion Lab-hybrid technology multithreaded architecture (JPL-HTMT) (49-220-85603-0-3950) and Rome Labs (F30602-97-C-0049).

E. Chuang was with the California Institute of Technology, Pasadena, CA 91125 USA. He is now with Sony Corporation, Tokyo 141-0001 Japan.

W. Liu and D. Psaltis are with the California Institute of Technology, Pasadena, CA 91125 USA.

J.-J. P. Drolet was with the California Institute of Technology, Pasadena CA 91125 USA. He is now with MicroDisplay Corporation, San Pablo, CA 94806 USA.

Publisher Item Identifier S 0018-9219(99)07837-8.

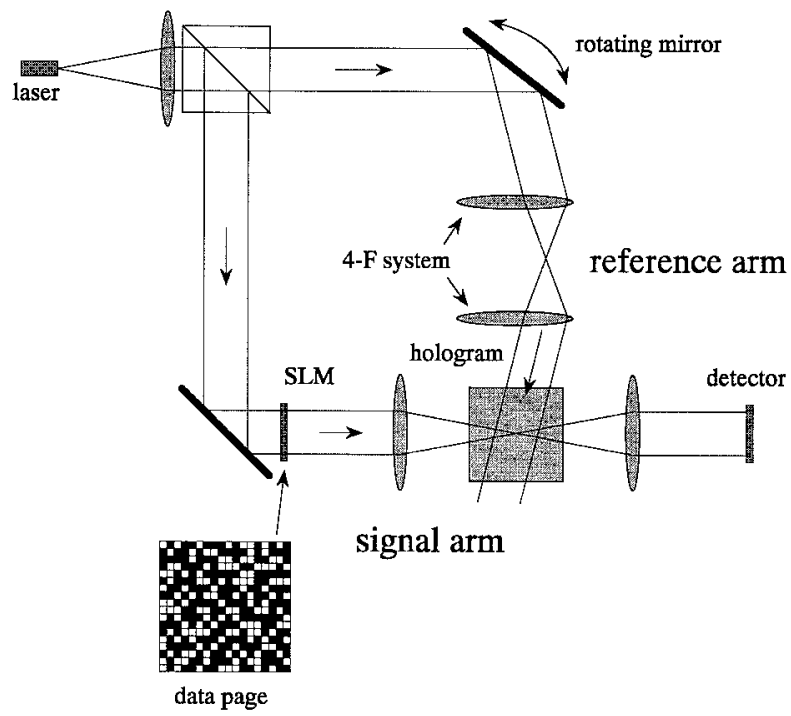

Fig. 1. Typical angle-multiplexed holographic memory.

Fig. 1 shows a typical angle-multiplexed holographic memory in the $90^{\circ}$ geometry. We can multiplex thousands of holograms within the same volume of the material by slightly changing the angle of the reference beam with each new data page, offering the potential of very high storage densities. Furthermore, holography has the inherent advantage of massive parallelism. Unlike conventional storage media such as magnetic hard disks and CD-ROM's which access only 1 bit at a time, each access of a holographic memory yields an entire data page-more than a megabit at a time.

In this paper we describe a holographic random access memory (HRAM) design that leads to the implementation of compact and inexpensive memory modules that can be used to construct large read-write memories. The potential place for such devices in the memory hierarchy is between magnetic disks and semiconductor memories (DRAM). Specifically, we believe that HRAM can become a competitive technology if optoelectronics technology can achieve the following three milestones in the next few 
years:

1) amall SLM and detector pixel sizes on the order of $1 \mu \mathrm{m}$;

2) high recording sensitivity of the holographic material with no more than $1 \mathrm{~J} / \mathrm{cm}^{2}$ to reach saturation;

3) inexpensive high spatial density laser diodes with at least $500 \mathrm{~mW}$ of output power in the near-infrared or red wavelength.

We will review the HRAM design and present experimental results from this architecture. We will estimate the performance that can be achieved with currently available technologies, and we justify the need to meet the milestones listed above in order for HRAM to become competitive. Specifically, if these developments take place, then HRAM can succeed as a memory that is less expensive than DRAM and faster than magnetic disks.

\section{Conjugate Readout Method}

Despite the high theoretical limit on the storage density of volume holographic storage (1 bit/cubic wavelength of material [17]), the practical implementation of holographic systems is often bulky due to the large space occupied by the various components that are necessary to provide the recording and readout mechanisms for the crystal. The system of Fig. 1 is fairly simple with a relatively small number of components, however the spacing requirements of the imaging lenses impose constraints on how closely these components can be placed. For example, assuming spatial light modulator (SLM) and detector array dimensions of 1 $\mathrm{cm}$ and high quality lenses with $F / \#=1$, the focal distance between the arrays, lenses, and crystal must also be at least $1 \mathrm{~cm}$. The system of Fig. 1 would then occupy a volume of approximately $6 \mathrm{~cm} \times 5 \mathrm{~cm} \times 1 \mathrm{~cm}$, which is 30 times larger than the volume of the recording material.

The reason we normally need to place lenses within the signal path is to undo the effects of diffraction. When we record a hologram of the signal beam diverging from the input SLM and reconstruct it with the original reference beam, we produce a virtual image of the input data page and thus require a lens to refocus it onto the detector array. We can eliminate the lens system between the SLM and detector array if we reconstruct a real image instead of a virtual one. One way to do this is to use phase conjugate readout [18]-[20] as illustrated in Fig. 2. Using this method, a hologram is recorded in the usual manner between the signal and reference beams, but the hologram is read out with the phase conjugate of the reference beam, propagating in the opposite direction as the one used for recording. This causes the signal reconstruction from the hologram to propagate back along the direction from which it originally came, reversing the original signal diffraction, and refocusing exactly at the plane of the SLM array. To generate the conjugate reference we may use a phaseconjugate mirror [19], or in the case of a planar reference beam, we may simply use a counter-propagating plane wave at the each angle.
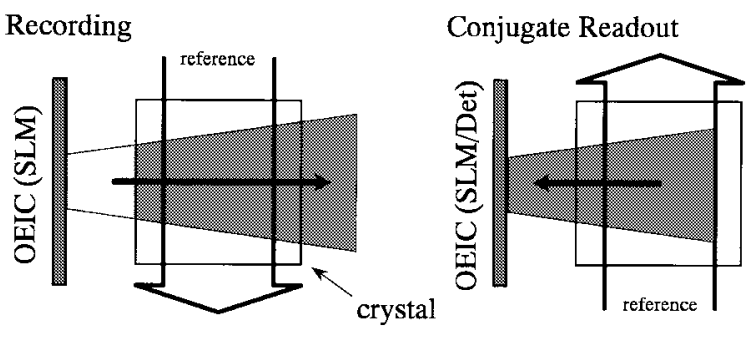

Conventional Readout

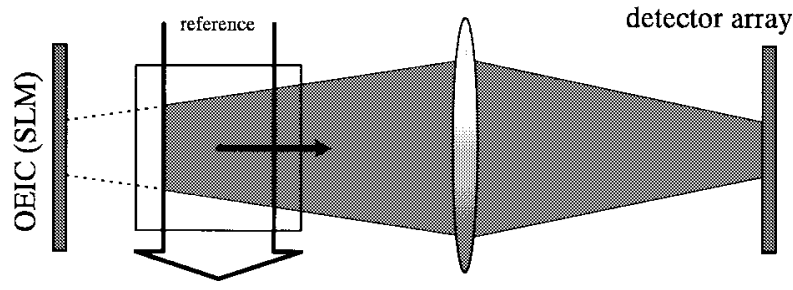

Fig. 2. Comparison of phase conjugate readout method with conventional readout using imaging lenses.

We compared experimentally the reconstructed image fidelity that can be obtained with conventional reconstruction using high-quality, custom-designed lenses to the image fidelity we get with the conjugate readout method. An SLM and detector array, each with pixel pitch of $24 \mu \mathrm{m}$, were used for these tests, allowing one-to-one matching of the SLM and detector pixels. Both methods yielded signal-to-noise ratio (SNR) values ranging from about 3.8 to 4.5 , verifying that the conjugate readout method produces results that can only be achieved with quality lenses, while using a much more compact and inexpensive optical system.

Conjugate readout eliminates the lenses and associated path lengths that are normally required in the signal path, but it requires that both the input and output devices be located on the same side of the crystal, as shown in Fig. 2. One approach is to employ a smart-pixel array that combines at each pixel the functions of a light modulator and a detector in a single optoelectronic integrated circuit (OEIC).

\section{DyNAMiC Hologram Refresher CHIP}

In the experimental demonstrations that we describe below we implemented smart-pixel arrays by merging liquid crystal and silicon technologies [21], [22]. Fig. 3(a) shows the cross section of our device [23]. It is composed of silicon circuitry overlaid with hybrid-aligned nematic liquid crystal [24], [25] and a glass cover plate with a transparent electrode. The circuitry contains a photodetector, a static memory element to hold the data that are read out or that are to be written, and liquid crystal driving circuitry. Each pixel contains an exposed metal pad on top of the silicon that is used to modulate field across the liquid crystal layer, which in turn modulates the polarization of the light reflected from the metal pad. As a result, with a properly oriented polarizer at the output, the reflectance from each SLM pixel can be turned on or off. 


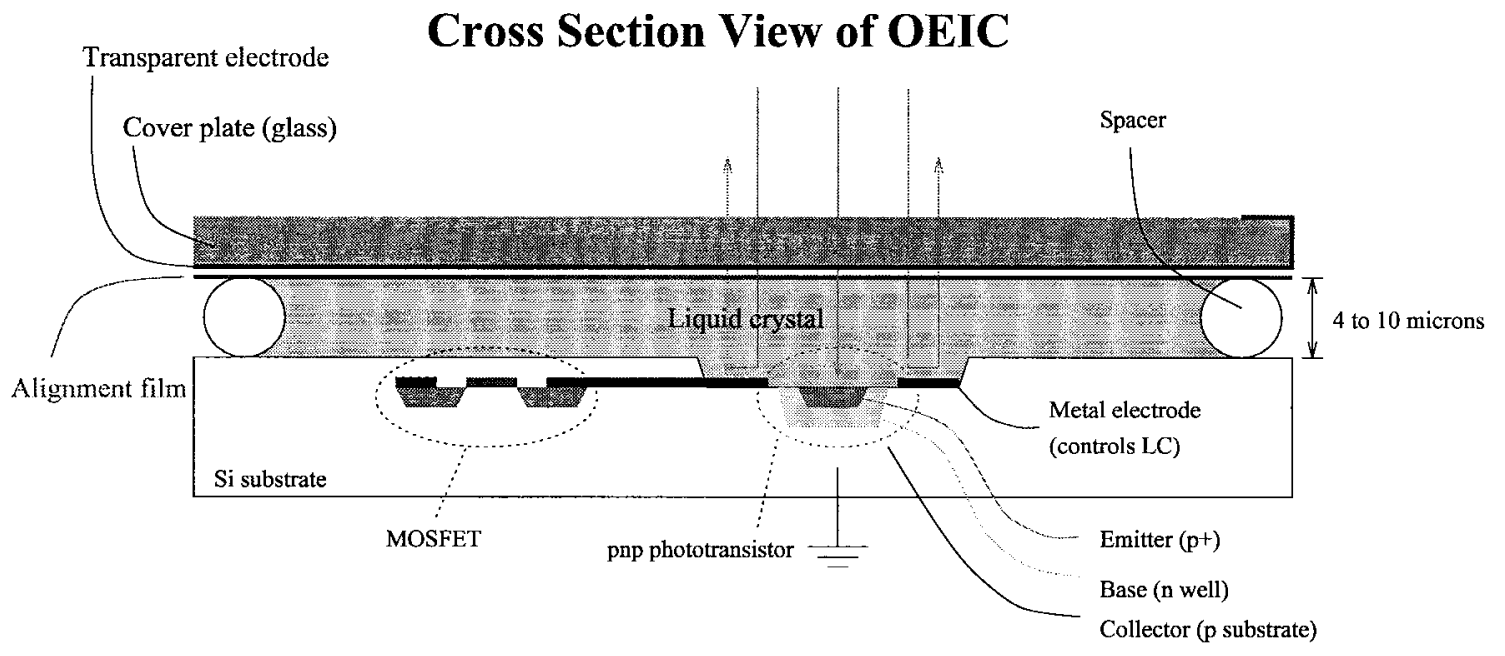

(a)

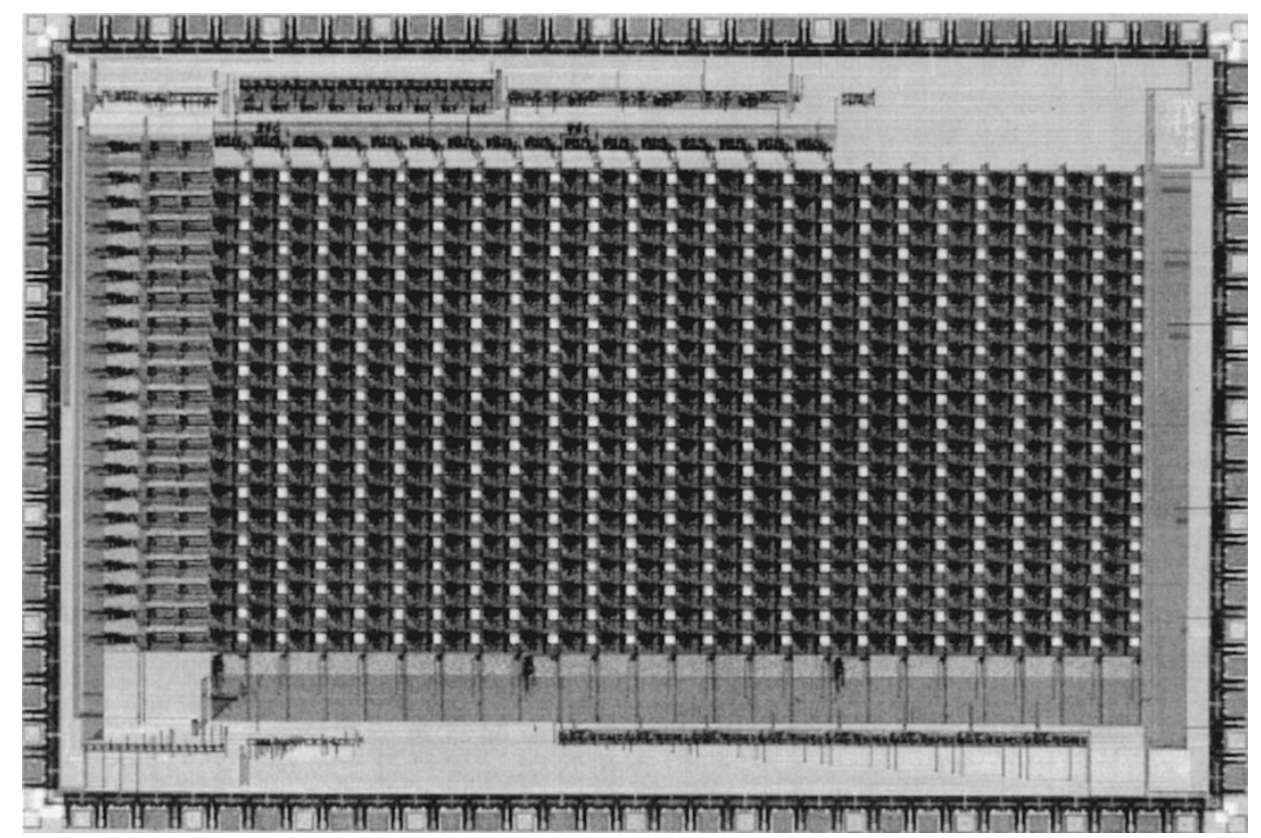

(b)

Fig. 3. (a) Cross-section diagram of dynamic hologram refresher chip and (b) picture of actual device array.

Fig. 3(b) shows a picture of the prototype array. It contains an array of $20 \times 24$ pixels which was designed to appear as a static random-access memory to a controlling microprocessor. The photodetector in each pixel is a PNP active-well-substrate structure. The pixel size in this prototype is $132 \mu \mathrm{m} \times 211 \mu \mathrm{m}$. The dimensions of the reflective SLM pads within each pixel is $49 \mu \mathrm{m}$ square, with $18 \mu \mathrm{m}$ square photodetector pads adjacently located. For reasons that will become apparent in the next section we refer to this device as the dynamic hologram refresher (DHR) chip.

\section{PERIODIC COPYING}

If we wish to use a holographic system as a rewritable memory, we must preserve the dynamic nature of the recorded gratings. For this purpose, photorefractive crystals (e.g., lithium niobate or barium titanate) are currently the most promising type of holographic media. Unfortunately, when a photorefractive crystal is used as the recording material, the recorded gratings decay when illuminated by the readout beam. One way to compensate for this is to use copying techniques [26]-[29] to periodically refresh the recorded holograms. Using this approach, stored holograms must be intermittently read out and rewritten into the memory in order to strengthen the gratings.

We conducted an experiment to test the periodic copying technique by building a holographic memory using the conjugate readout method and the DHR chip. A schematic and photograph of the experimental setup is shown in Fig. 4. The photorefractive medium was a cube of $\mathrm{BaTiO}_{3}$, cut $30^{\circ}$ with respect to its $c$ axis as shown in the figure. We used the DHR chip to serve as both input SLM and output detector. We used light from an argon laser at a wavelength of $488 \mathrm{~nm}$ for these experiments. The crystal was mounted on a rotation stage for angular multiplexing. We recorded 25 


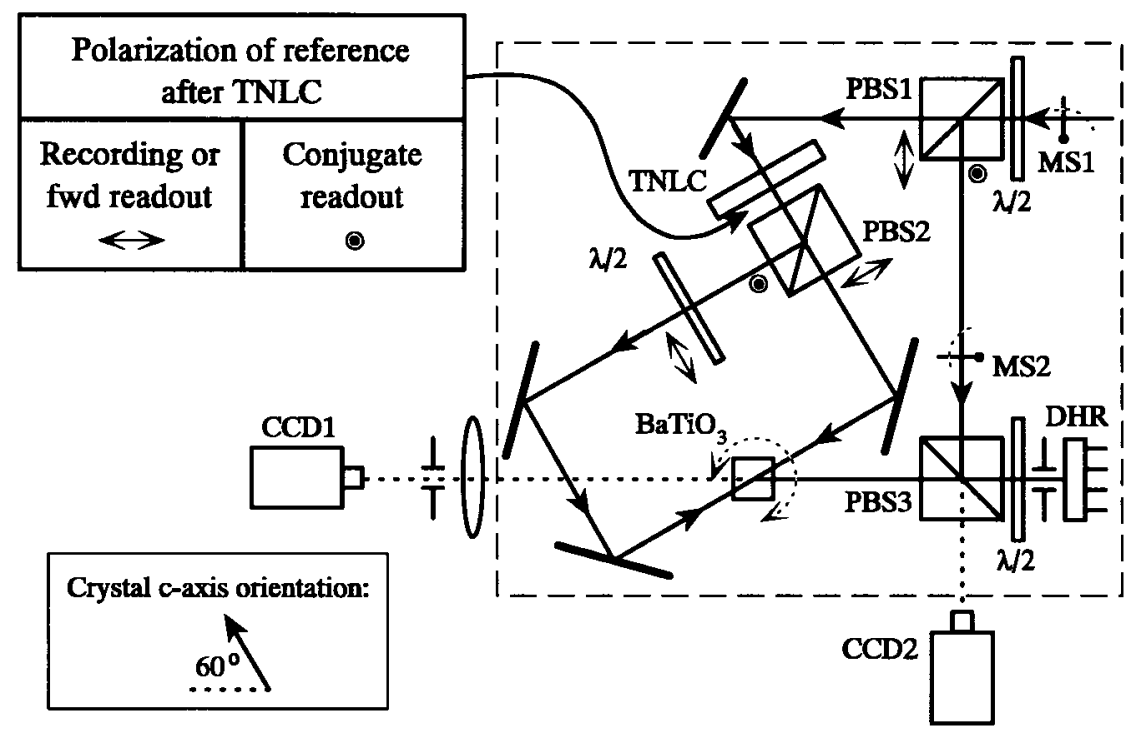

(a)

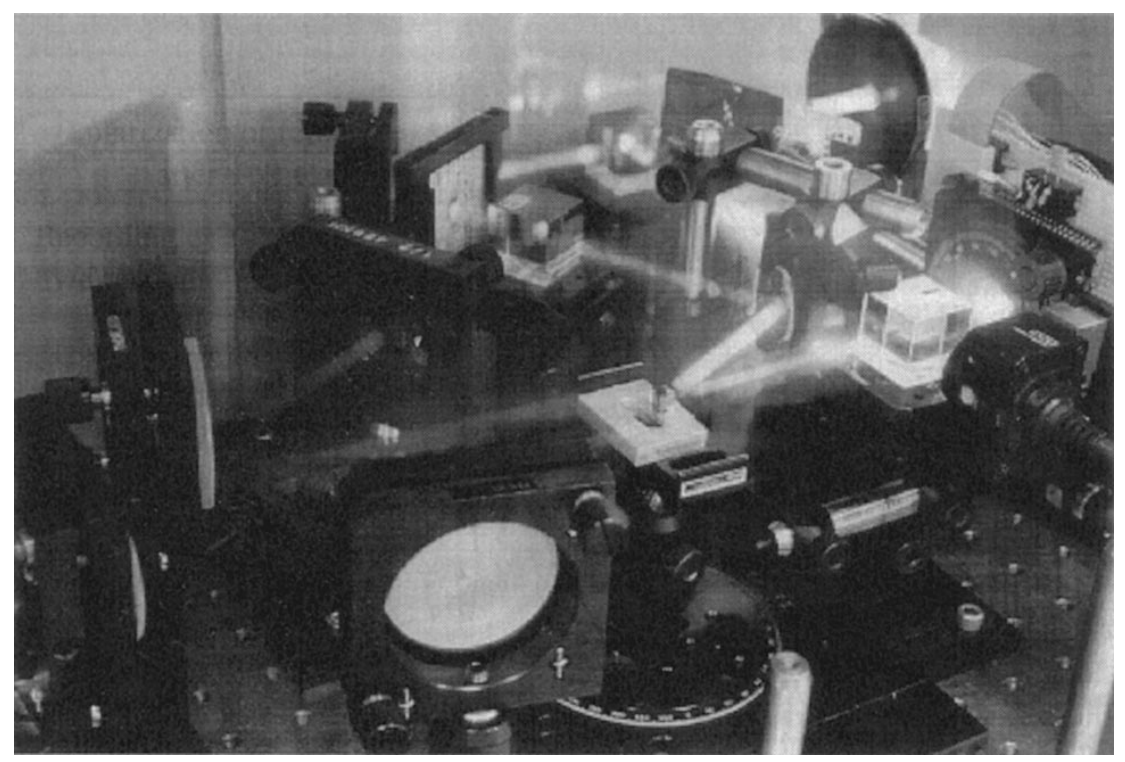

(b)

Fig. 4. (a) Schematic of experimental setup for conjugate readout with periodic copying in the transmission geometry and (b) a photograph of the setup.

holograms (the letters "CIT" displayed on the DHR). Each hologram was initially recorded for $4 \mathrm{~s}$ with the input data page. Upon completion of the recording cycle, we immediately returned to the first hologram to begin the refresh cycle. The purpose of the refreshing is to restore the strength of the holograms to their original recorded strength after they decay when they are read out. To refresh each hologram, the stored hologram was read by the conjugate beam, and these retrieved data were used to rewrite the hologram for the same recording time as the initial recording, $4 \mathrm{~s}$. This refresh was repeated for each of the 25 holograms, and the entire refresh cycle was repeated for 100 cycles.

Fig. 5 shows the diffraction efficiencies measured from the experiment for all 25 holograms. The curves for all of the holograms are superimposed in this graph to demonstrate the consistency among the hologram grating strengths. A few sample reconstructions from this experiment are shown in Fig. 6. Fig. 6(a) shows a sample conjugate reconstruction after the initial recording; Fig. 6(b)-(d) shows conjugate reconstructions of holograms $\# 1$, \#13, and \#25 at the end of the 100 refreshings. The conjugate diffraction efficiency was measured to be $77 \%$ of the forward diffraction efficiency in this experiment.

Results of analyzing these images for SNR and probability of error are summarized in Table 1. Both visually and analytically we observed no appreciable loss in image quality from the refresh operations. The higher SNR and lower probability of error at the end of the experiment is consistent with the fact that after 100 refreshes, all of the holograms are well into the steady state region where the diffraction efficiencies are significantly higher than after the initial recording. Also, among the three holograms examined at the end of the experiment, the holograms toward the end of the cycle yield better values because their 


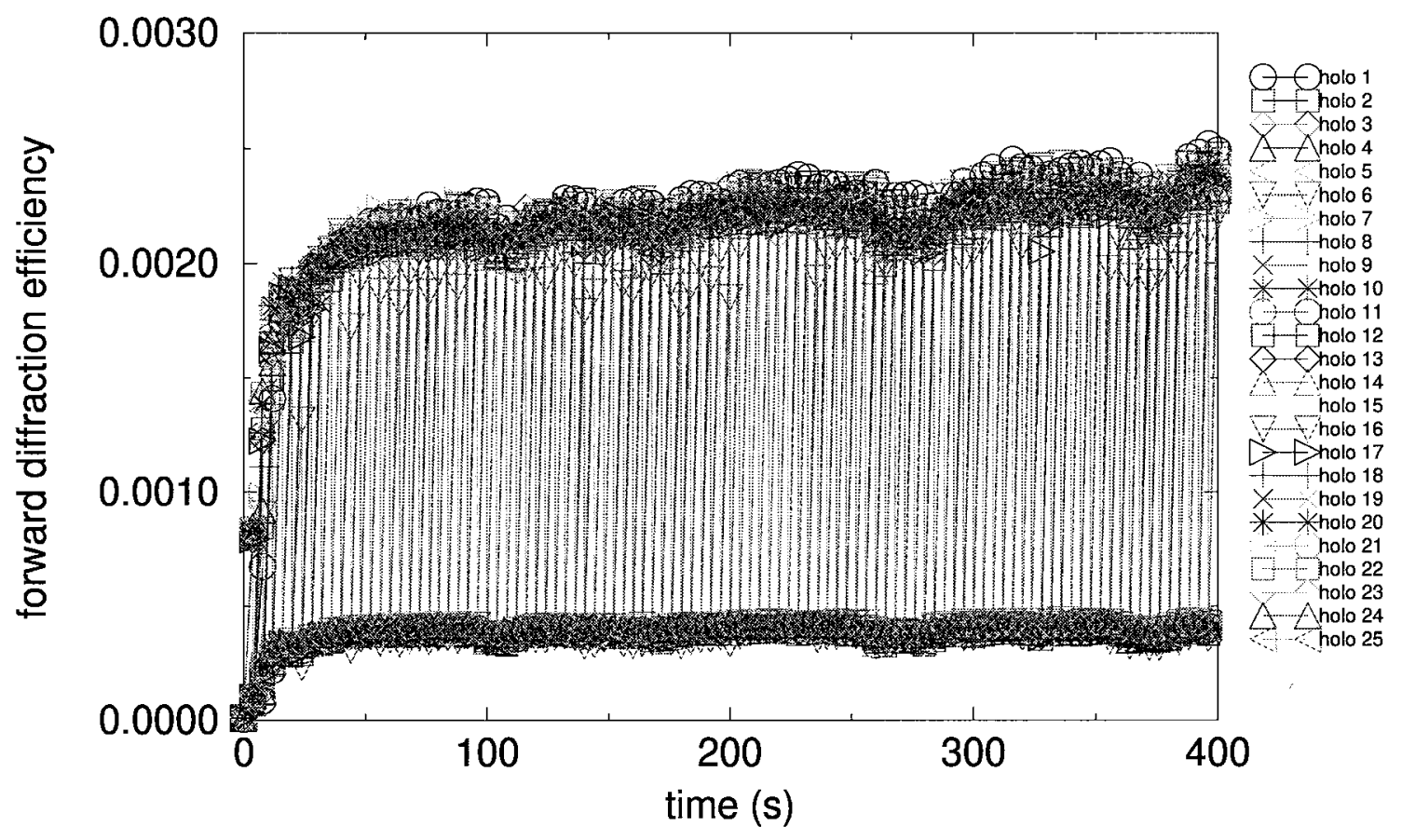

Fig. 5. Evolution of diffraction efficiencies of 25 angle-multiplexed holograms over 100 cycles in the transmission geometry.

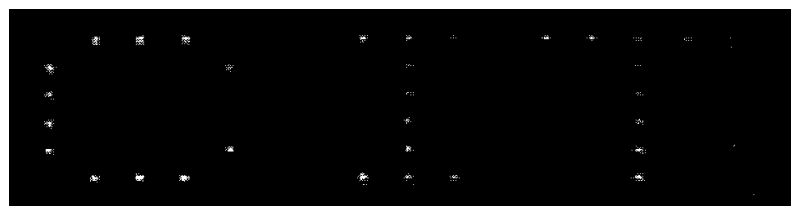

(a)

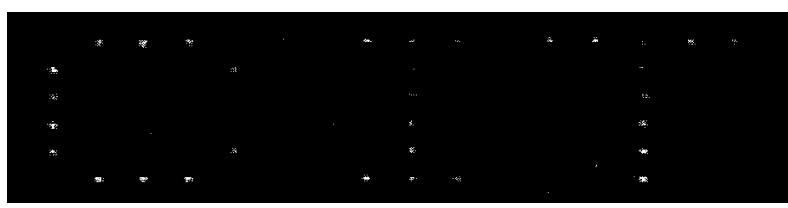

(b)

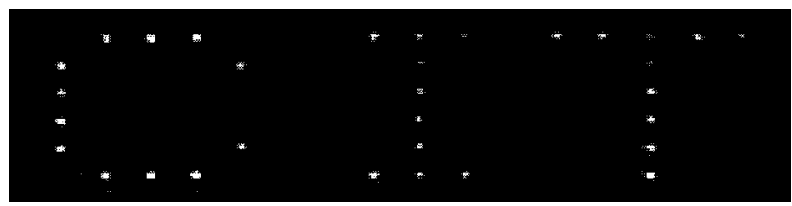

(c)

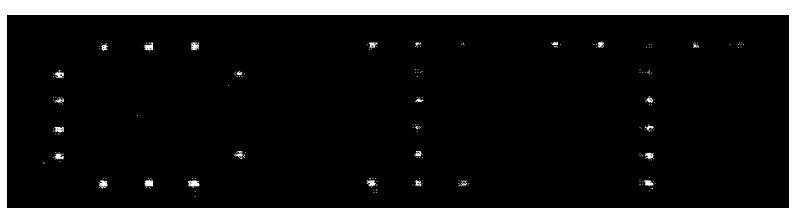

(d)

Fig. 6. Sample reconstructions from 25-hologram experiment: (a) after initial recording; (b) hologram \#1; (c) hologram \#13; and (d) hologram \#25 after 100 refreshes.

diffraction efficiencies are higher, having been the most recently refreshed and thus the strongest. No errors were detected in any pixel in any hologram during the course of the experiment.
Table 1

SNR and Probability of Error Corresponding to the Images Shown in Fig. 6

\begin{tabular}{l|c|c}
\hline Image & SNR & PE \\
\hline \hline Conjugate reconstruction, 1 cycle & $3.94: 1$ & $8.2 \times 10^{-4}$ \\
\hline Hologram \#1, 100 cycles & $4.28: 1$ & $1.0 \times 10^{-4}$ \\
\hline Hologram \#13,100 cycles & $4.69: 1$ & $5.3 \times 10^{-5}$ \\
\hline Hologram \#25, 100 cycles & $5.03: 1$ & $2.9 \times 10^{-5}$ \\
\hline
\end{tabular}

\section{COMPACT FAST-ACCESS ARCHITECTURE}

While conjugate readout eliminates the lenses in the signal path of the memory system, we still require a compact design to rapidly deflect the reference beam for multiplexing purposes. The $4-F$ system shown in Fig. 1, while reliable, is bulky and slow due to the limited mechanical speed of the rotating mirror.

With the recent development of compact laser emitters, such as laser diodes and vertical-cavity surface-emitting laser (VCSEL) devices [30], [31], it has become feasible to consider the possibility of incorporating arrays of hundreds of microscopic laser sources in a holographic memory. We can then design a system in which each angle multiplexed hologram is addressed by a dedicated laser source. This architecture is shown in Fig. 7, where the signal laser is coherent with the reference laser array. A Fourier transforming lens is used to convert the spatial shifts between the reference laser elements into angularly offset plane waves incident on the crystal. In this implementation, the time it takes to produce the proper read-out reference beam 


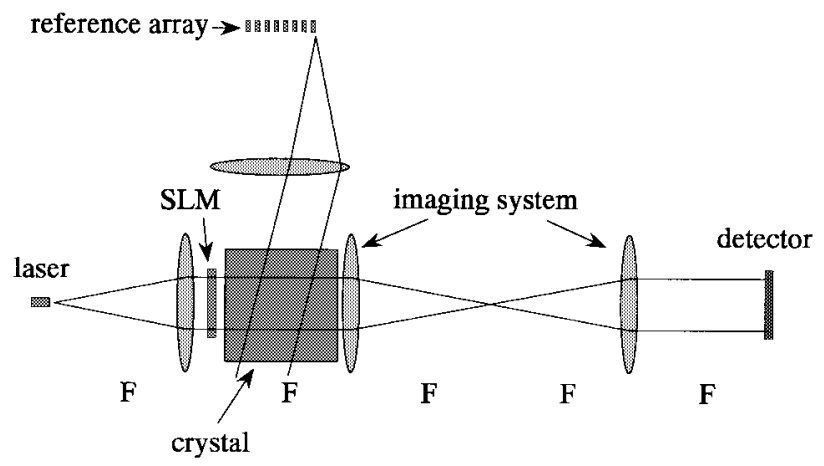

Fig. 7. Use of a laser array in the reference arm of an angle multiplexed memory for fast page access.

is determined by the switching time of the laser sources, which is in the nanosecond regime. Using a 1-cm thick crystal and a wavelength of $630 \mathrm{~nm}$, the first null of the angular selectivity function occurs at an angular spacing of $0.0036^{\circ}$. Using a lens with a focal length of $2 \mathrm{~cm}$ would require the laser elements to be placed only $1.3 \mu \mathrm{m}$ apart to produce this angular separation. In practice, the separation is $10 \mu \mathrm{m}$ or more in order to reduce interpage crosstalk while also making the array easier to fabricate.

This approach is also compatible with the conjugate readout method as shown in Fig. 8(a). The properly aligned laser array elements have the wavelength deviation much smaller than the hologram wavelength selectivity $\left(10^{-5} \lambda\right.$ for 1-cm thick crystal). With a mirror placed on the opposite face of the crystal such that it lies at the focus of the Fourier transforming lens, the proper conjugate beam can be generated with the symmetrically opposite laser source and overlap the original writing beam path inside the crystal. A beamsplitter must also be introduced to accommodate both the SLM and detector devices. The combination of conjugate readout in the signal beam path and laser diode arrays in the reference beam path results in a very compact holographic memory module with fast access. It is not completely lensless, since one lens still remains in the system, but such a lens would be required to collimate the laser source in any optical system that uses plane waves. This module design can be easily modified to accommodate a smart pixel array, such as the DHR chip, as shown schematically in Fig. 8(b) and visually by the model in Fig. 9.

\section{A. Readout}

Since the laser diode array discussed in the previous section allows us to switch between multiplexed data pages with negligible delay (on the order of nanoseconds), the random access time and the readout rate become limited by the required integration time of the detector. We can write the integration time as

$$
\text { Detector integration time }=\frac{N_{e} h \nu N^{2}}{\left(\frac{M / \#}{M}\right)^{2} P_{i}}
$$

where $N_{e}$ is the number of electrons per pixel that we need to integrate for the given detector sensitivity and level of background noise, $h$ is Planck's constant (6.63 $\left.\times 10^{-34} \mathrm{~J} \cdot \mathrm{s}\right), \nu$ is the light frequency, $N^{2}$ is the total number of pixels in the detector array, $M / \#$ is the system metric [32] of the holographic medium, $M$ is the number of multiplexed holograms, and $P_{i}$ is the incident readout power. For example, if we use a crystal of $M / \#=10$ to record 500 holograms of a $1000 \times 1000$ pixel array, and we read out with $100 \mathrm{~mW}$ of laser power, requiring 300 electrons per pixel, the integration time, and hence the random access time, would be $2.4 \mu \mathrm{s}$. This corresponds to a sustained readout transfer rate, from the hologram to the silicon detectors, of 53 Gbytes/s.

\section{B. System Volume Density}

An analysis of the system storage density (including the recording medium and all the optical components) of the holographic memory module of Fig. 8(a) shows that the module storage density peaks at about $40 \mathrm{Mb} / \mathrm{cm}^{3}$ for an optimum pixel size of $5 \mu \mathrm{m}$ [33]. There is an optimum pixel size because as the pixel size decreases, the light in the signal path spreads more due to diffraction, causing us to use larger apertures for the crystal and beamsplitters.

A more aggressive concept for minimizing the volume is shown in Fig. 10. This design relies on total internal reflection to contain the beam diffraction within the boundaries of the module, so that the optical elements can be made close to the size of the SLM array, as far as the size of the laser array is relatively much smaller than the SLM. Preliminary experiments indicate that accurate recordings are obtained using the internally reflected light. In this case, the system density can be raised to the order of 2 $\mathrm{Gb} / \mathrm{cm}^{3}$, if SLM pixel sizes fall to $1 \mu \mathrm{m}$. At this density, a gigabyte of data could be stored in a single module with a volume of $1 \times 2 \times 2 \mathrm{~cm}^{3}$. The challenges in achieving such high densities are several: development of SLM and detectors with $1-\mu \mathrm{m}$ pixels; development of laser array with high spatial density; designing the optical system so that we have uniform illumination throughout; and further characterization of the performance of the module when the light is allowed to undergo total internal reflection.

\section{Recording Rate}

We can write the recording rate of the memory module as

$$
\text { Recording rate }=\frac{N^{2} I S L p}{(M / \#) / M}
$$

where $N^{2}$ is the total number of pixels per data page, $I$ is the incident recording intensity, $S$ is the sensitivity per unit length of the recording medium, $L$ is the crystal thickness, and $p$ is the light efficiency of the SLM. Again assuming a crystal of $M / \#=10$ to record 500 holograms of a $1000 \times 1000$ pixel array, with $I=100 \mathrm{~mW} / \mathrm{cm}^{2}$, $S=0.1 \mathrm{~cm} / \mathrm{J}, L=1 \mathrm{~cm}$, and $p=50 \%$, we obtain a recording rate of $31 \mathrm{kbytes} / \mathrm{s}$. This is typical for experiments currently performed. Increasing the recording rate to make it comparable to the readout rate is highly desirable for a practical system. We will discuss possible methods for achieving this goal later on. 


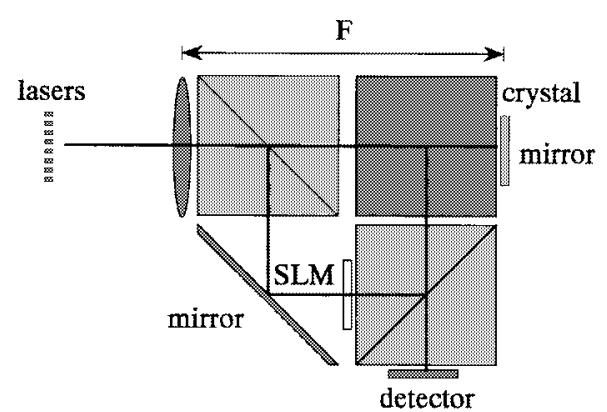

(a)

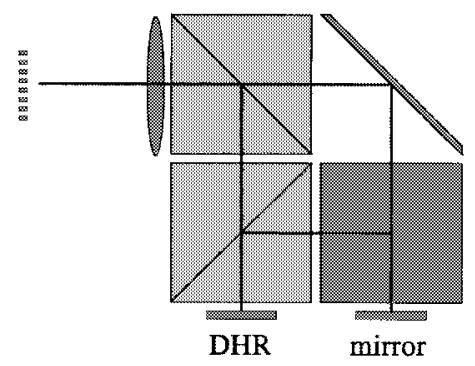

(b)

Fig. 8. Variations of compact memory module incorporating (a) separate SLM and detector devices or (b) using a smart pixel array (DHR) combining SLM and detector functions.

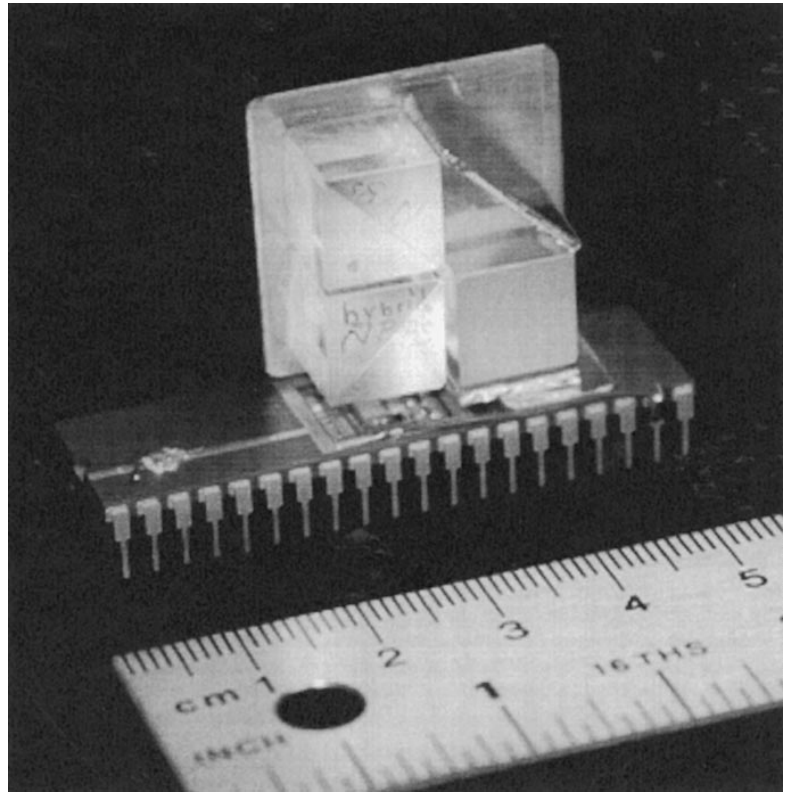

Fig. 9. Model of the HRAM module with the DHR chip.

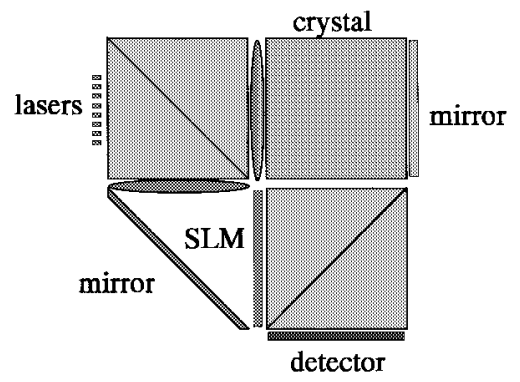

Fig. 10. Variation of compact memory module for minimum volume.

\section{Cost}

The cost is perhaps the most important metric for accessing the commercialization prospects of HRAM. We will compare the costs of HRAM and DRAM with reference to Fig. 11. We can think of HRAM as a holographic module which sits on top of a page of DRAM. The ability of the HRAM to multiplex holograms essentially allows us to store $M$ DRAM data pages, hence saving us the cost of fabricating $M-1$ additional DRAM pages in silicon. However, it is not quite that simple. First, the silicon device

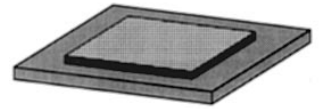

\section{$\mathrm{N}$ cells \\ 1 page Capacity: $\mathbf{N}$}

(a)

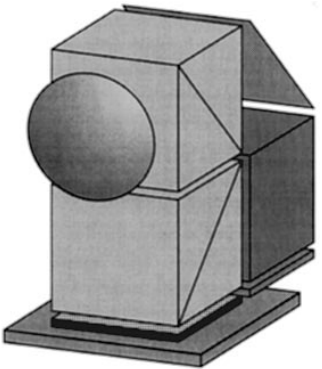

M pages

Capacity: N M / R

(b)
N/R pixels/page

Fig. 11. Model for cost comparison between HRAM and DRAM.

Table 2

Estimated Cost of Components in the Holographic Memory Module, Assuming Production in Large Quantities

\begin{tabular}{l|l}
\hline Component & Estimated cost \\
\hline \hline LiNbO $_{3}\left(1 \mathrm{~cm}^{3}\right)$ & $\$ 10$ \\
\hline Liquid crystal & $\$ 5$ \\
\hline Beamsplitters and lens & $\$ 6$ \\
\hline Silicon $\left(1 \mathrm{~cm}^{2}\right)$ & $\$ 115$ \\
\hline Laser diode array $(500)$ & $\$ 25-100$ \\
\hline \hline Total: & $\$ 161-236$ \\
\hline
\end{tabular}

in the HRAM is not really a DRAM page, but rather the DHR chip described earlier or an SLM/detector pair. Because of the necessity of fabricating SLM and detector pixels (either in the same OEIC or in two separate devices), the page density of the DHR will be less than that of a true DRAM. We call this ratio of the page densities $R>1$. Moreover, the cost of the holographic module also includes the cost of the optical elements $\left(C_{\mathrm{Opt}}\right)$ and laser diode array $\left(C_{\mathrm{LD}}\right)$, in addition to that of the silicon $\left(C_{\mathrm{Si}}\right)$. The projected costs of the optical elements (assuming production in large quantities) are summarized in Table 2 . We assume the silicon cost to be purely based on area, and therefore 
Table 3

Comparison Between DRAM and HRAM

\begin{tabular}{l|c|c|c}
\hline & HRAM(current) & DRAM(2006) & HRAM (target) \\
\hline \hline Access time & $2.4 \mu \mathrm{s}$ & $10-40 \mathrm{~ns}$ & $530 \mu \mathrm{s}$ \\
\hline Recording rate & $31 \mathrm{kB} / \mathrm{s}$ & $10 \mathrm{~GB} / \mathrm{s}$ (pin limited) & $1.0 \mathrm{~GB} / \mathrm{s}$ \\
\hline Readout rate & $53 \mathrm{~GB} / \mathrm{s}$ (optical limit) & & $24 \mathrm{~GB} / \mathrm{s}$ (optical limit) \\
& $10 \mathrm{~GB} / \mathrm{s}$ (pin limited) & $10 \mathrm{~GB} / \mathrm{s}$ (pin limited) & $10 \mathrm{~GB} / \mathrm{s}$ (pin limited) \\
\hline Cost & N.A. & $\$ 0.40 / \mathrm{MB}$ & $\$ 0.04 / \mathrm{MB}$ \\
\hline
\end{tabular}

will be identical to that for an equal-sized DRAM. The cost of the laser array is not well known at this time, since large arrays have not yet been produced for visible wavelengths; however, we estimate the cost to be in the range of $\$ 25-\$ 100$ per array.

We can then write the cost-per-bit ratio of the HRAM to DRAM as

$$
\text { Cost Ratio }=\frac{C_{\mathrm{Si}}+C_{\mathrm{Opt}}+C_{\mathrm{LD}}}{C_{\mathrm{Si}}} \cdot \frac{R}{M} .
$$

For current commercial SLM's and detector arrays, the smallest available pixel pitch is on the order of $4 \mu \mathrm{m}$, with the spacing of DRAM cells at $1 \mu \mathrm{m}$, leading to a value of $R=16$. Hence, in order for HRAM to have a cost advantage over DRAM by a factor of ten or more, we need to record at least 200-300 holograms in each HRAM module. Since this can be readily achieved, cost emerges as the major competitive advantage of HRAM.

\section{ROADMAP FOR A COMPETITIVE HRAM TECHNOLOGY}

From the preceding discussion, we can summarize the current parameters for the HRAM system as shown in Table 3. For comparison, we also show the specifications projected for DRAM by the year 2006. DRAM access times should fall to 10-40 ns; the DRAM transfer rates can reach 10 Gbytes/s, assuming for example 800 pins, each with a bandwidth of $100 \mathrm{MHz}$. The cost is projected to be $\$ 0.40 /$ Mbyte [34]. Although the holographic readout rate of the HRAM system is nominally 53 Gbytes/s, the fact that its readout interface is through silicon (the DHR) limits the transfer rate to that of DRAM, 10 Gbytes/s.

Presently, the greatest challenge for the HRAM is to raise its recording rate by several orders of magnitude. To achieve this, we must rely in part on improvements in SLM technology to bring the pixel sizes down to 1 $\mu \mathrm{m}$. This will allow us to increase the size of each data page to $10000 \times 10000$ pixels while still holding the array size to about $1 \mathrm{~cm}^{2}$. By increasing the page size in this way, we immediately gain two orders of magnitude in the sustained recording rate due to the increased parallelism. Experimentally, we have used a mask fabricated with $e-$ beam lithography to record and reconstruct data pages with $1-\mu \mathrm{m}$ pixels holographically with good image fidelity. Fig. 12 shows the results from an experiment in which

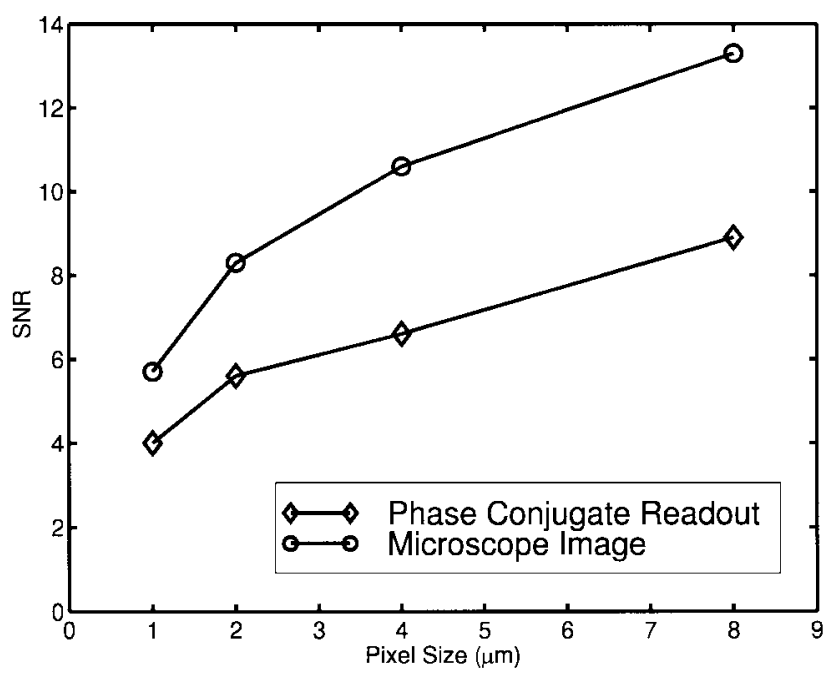

Fig. 12. SNR versus pixel size measured for both direct imaging and for conjugate hologram reconstructions. At the target value of $1-\mu \mathrm{m}$ pixels, the $\mathrm{SNR}=4$.

we measured the SNR from the conjugate reconstruction of various pixel sizes. The reconstruction of $1-\mu \mathrm{m}$ pixels yielded values of SNR $=4$.

Reducing the pixel sizes to $1 \mu \mathrm{m}$ is not only necessary for raising the recording rate, but also for maintaining the cost advantage of HRAM over DRAM. By 2006, the DRAM cell pitch is expected to fall to $0.2 \mu \mathrm{m}$ [34]. By bringing the SLM pixel pitch down to $1 \mu \mathrm{m}$, we can hold the factor $R$ in (3) at 25, and still beat the cost of DRAM by an order of magnitude by recording only $350-500$ holograms.

Because the HRAM readout rate is limited by the electronic transfer rate out of the detector chip, we can afford to give up some readout speed in favor of increasing the recording speed. We do this by intentionally reducing the strength of the holograms so that we can record with shorter exposures, at the cost of increasing the detector integration time. In (1) and (2), this is equivalent to recording in a medium with lower $M / \#$, but without sacrificing sensitivity. Unfortunately, as we increase the required integration time, we increase at the same time the random access time of the memory. In order to maintain an advantage of at least an order of magnitude over magnetic disks in random access time, we can only afford to increase the integration time to several hundreds of microseconds. 
Other opportunities for increasing the recording rate can arise from improvements in laser output powers or from improving the sensitivity of the recording materials. Compact laser arrays with outputs of $500 \mathrm{~mW}$ per emitter may be possible by 2006 , or if not, we may consider sharing a larger, more powerful tunable laser among multiple HRAM modules. Increasing material sensitivity presents more of a challenge. The sensitivity of $\mathrm{LiNbO}_{3}: \mathrm{Fe}$, by far the most commonly used recording material today, is typically around $0.02 \mathrm{~cm} / \mathrm{J}$ in the $90^{\circ}$ geometry. In order to get recording rates on the order of 1 Gbytes/s, we must find ways to boost the material sensitivity to about $1 \mathrm{~cm} / \mathrm{J}$ by improving lithium niobate's properties. For instance, switching to transmission geometry and increasing the doping level results in substantial increases in $M / \#$, which can be traded for better sensitivity as we discussed previously. Alternatively, we can switch to materials such as barium titanate which we measured to have $S=0.55$ $\mathrm{cm} / \mathrm{J}$ in the $90^{\circ}$ geometry. Even higher sensitivities are possible in the transmission geometry. However, this is a relatively untested material compared to lithium niobate and much more expensive at present.

\section{CONCLUSION}

In order to develop a competing HRAM technology, three main challenges must be met: reducing pixel size to $1 \mu \mathrm{m}$; producing arrays of high-power laser diodes; and increasing the sensitivity of holographic recording media. Each of these tasks is difficult, but if they can be achieved in the next few years, then the projected HRAM performance levels shown in Table 3 become feasible. These values assume an array size of $10000 \times 10000$ pixels, 500 holograms recorded to diffraction efficiencies in a $1-\mathrm{cm}$ thick material with effective $M / \#=3$ and 2 $\mathrm{cm} / \mathrm{J}$ sensitivity, laser power of $500 \mathrm{~mW}$, and 300 electrons required for detection. Attaining these goals will position the HRAM as a viable alternative memory technology to magnetic storage, offering performance that is at least one order of magnitude better in terms of random access and transfer rate than magnetic hard disks, and no more than one tenth the cost compared to fabricating an equivalent memory in DRAM.

\section{ACKNOWLEDGMENT}

The authors would like to thank G. Barbastathis, X. Wang, and A. Adibi for helpful discussions.

\section{REFERENCES}

[1] D. Psaltis and F. Mok, "Holographic memories," Scientific Amer., vol. 273, pp. 70-76, Nov. 1995.

[2] J. H. Hong, I. McMichael, T. Y. Chang, W. Christian, and E. G. Paek, "Volume holographic memory-systems: Techniques and architectures," Opt. Eng., vol. 34, pp. 2193-2203, Aug. 1995.

[3] J. F. Heanue, M. L. Bashaw, and L. Hesselink, "Volume holographic storage and retrieval of digital data," Science, vol. 265, pp. 749-752, Aug. 1994.

[4] A. Pu and D. Psaltis, "High-density recording in photopolymerbased holographic 3-dimensional disks," Appl. Opt., vol. 35, pp. 2389-2398, May 1996
[5] D. Psaltis and G. W. Burr, "Holographic data-storage," Computer, vol. 31, pp. 52-60, Feb. 1998

[6] P. J. van Heerden, "A new optical method of storing and retrieving information," Appl. Opt., vol. 2, no. 4, pp. 387-392, 1963.

[7] F. H. Mok, "Angle-multiplexed storage of 5000 holograms in lithium niobate," Opt. Lett., vol. 18, no. 11, pp. 915-917, 1993.

[8] D. Psaltis, D. Brady, X. G. Xu, and S. Lin, "Holography in artificial neural networks," Nature, vol. 343, no. 6256, pp. 325-330, 1990

[9] F. T. S. Yu, S. Wu, A. W. Mayers, and S. Rajan, "Wavelength multiplexed reflection matched spatial filters using $\mathrm{LiNbO}_{3}$," Opt. Commun., vol. 81, no. 6, pp. 343-347, 1991.

[10] G. A. Rakuljic, V. Leyva, and A. Yariv, "Optical data storage by using orthogonal wavelength-multiplexed volume holograms," Opt. Lett., vol. 17, no. 20, pp. 1471-1473, 1992.

[11] J. T. LaMacchia and D. L. White, "Coded multiple exposure holograms," Appl. Opt., vol. 7, no. 1, pp. 91-94, 1968.

[12] T. F. Krile, R. J. Marks, II, J. F. Walkup, and M. O. Hagler, "Holographic representations of space-variant systems using phase-coded reference beams," Appl. Opt., vol. 16, no. 12, pp. 3131-3135, 1977

[13] V. N. Morozov, "Theory of holograms formed using a coded reference beam," Soviet J. Quantum Electron., vol. 7, no. 8, pp. 961-964, 1977.

[14] K. Curtis, A. Pu, and D. Psaltis, "Method for holographic storage using peristrophic multiplexing," Opt. Lett., vol. 19, no. 13, pp. 993-994, 1994.

[15] A. Pu, K. Curtis, and D. Psaltis, "A new method for holographic data storage in photopolymer films," in Nonlinear Optics: Materials, Fundamentals, and Applications. Piscataway, NJ: IEEE/Lasers and Electro-Optics Soc. OSA, 1994, pp. 433-435.

[16] G. Barbastathis, M. Levene, and D. Psaltis, "Shift multiplexing with spherical reference waves," Appl. Opt., vol. 35, no. 14, pp. 2403-2417, 1996.

[17] P. J. van Heerden, "Theory of optical information storage in solids," Appl. Opt., vol. 2, no. 4, pp. 393-400, 1963.

[18] J.-J. P. Drolet, G. Barbastathis, J. S. Patel, and D. Psaltis, "Liquid crystal devices for volume holographic memories," in Proc. OSA Annu. Meeting, Portland, OR, Sept. 1995.

[19] J.-J. P. Drolet, G. Barbastathis, and D. Psaltis, "Optoelectronic interconnects and packaging," in SPIE Critical Rev., vol. CR62, pp. 106-131, 1996

[20] Z. O. Feng and K. Sayano, "Compact read-only memory with lensless phase-conjugate holograms," Opt. Lett., vol. 21, pp. 1295-1297, Aug. 1996.

[21] L. K. Cotter, T. J. Drabik, R. J. Dillon, and M. A. Handschy, "Ferroelectric-liquid-crystal/silicon-integrated-circuit spatial light modulator," Opt. Lett., vol. 15, pp. 291-293, Mar. 1990.

[22] K. M. Johnson, D. J. McKnight, and I. Underwood, "Smart spatial light modulators using liquid crystal on silicon," IEEE J. Quantum Electron., vol. 29, pp. 699-714, Feb. 1993.

[23] J. Drolet, G. Barbastathis, J. Patel, and D. Psaltis, "Liquid crystal devices for volume holographic memories," in Proc. OSA Annu. Meeting, Sept. 1995.

[24] J. P. Drolet, J. Patel, K. G. Haritos, W. Xu, A. Scherer, and D. Psaltis, "Hybrid-aligned nematic liquid-crystal modulators fabricated on VLSI circuits," Opt. Lett., vol. 20, pp. 2222-2224, Nov. 1995

[25] J.-J. P. Drolet, E. Chuang, G. Barbastathis, and D. Psaltis, "Compact, integrated dynamic holographic memory with refreshed holograms," Opt. Lett., vol. 22, pp. 552-554, Apr 1997.

[26] D. Brady, K. Hsu, and D. Psaltis, "Periodically refreshed multiply exposed photorefractive holograms," Opt. Lett., vol. 15, pp. 817-819, July 1990.

[27] H. Sasaki, Y. Fainman, J. Ford, Y. Taketomi, and S. Lee, "Dynamic photorefractive optical memory," Opt. Lett., vol. 16, pp. 1874-1876, Dec. 1991.

[28] S. Boj, G. Pauliat, and G. Roosen, "Dynamic holographic memory showing readout, refreshing, and updating capabilities," Opt. Lett., vol. 17, pp. 438-440, Mar. 1992.

[29] Y. Qiao and D. Psaltis, "Sampled dynamic holographic memory," Opt. Lett., vol. 17, pp. 1376-1378, Oct. 1992.

[30] J. L. Jewell, K. F. Huang, K. Tai, Y. H. Lee, R. J. Fischer, S. L. Mccall, and A. Y. Cho, "Vertical cavity single quantum well laser," Appl. Phys. Lett., vol. 55, no. 5, pp. 424-426, 1989. 
[31] W. W. Chow, K. D. Choquette, M. H. Crawford, K. L. Lear, and G. R. Hadley, "Design, fabrication, and performance of infrared and visible vertical-cavity surface-emitting lasers," IEEE $J$. Ouantum Electron., vol. 33, pp. 1810-1824, Oct. 1997.

[32] D. Psaltis, D. Brady, and K. Wagner, "Adaptive optical networks using photorefractive crystals," Appl. Opt., vol. 27, no. 9, pp. 1752-1759, 1988.

[33] E. Chuang, "Methods and architecture for rewritable holographic memories," Ph.D. dissertation, California Inst. Technol., Pasadena, 1998.

[34] "The national technology roadmap for semiconductors," Semiconduct. Industry Assoc., San Jose, CA, Tech. Rep., 1997.

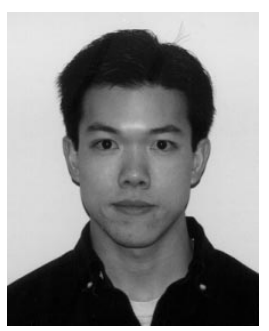

Ernest Chuang was born in Los Angeles, CA, in 1970. He received the B.S. degree in electrical engineering and computer science from the University of California, Berkeley, in 1992 and the M.S. and Ph.D. degrees in electrical engineering from the California Institute of Technology (CIT) in 1993 and 1998, respectively.

His research thesis at CIT was on rewritable holographic memories. In 1998, he joined Sony Corporation, Tokyo, Japan, where he is involved with research in optical data storage techniques.

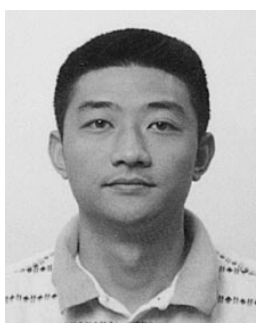

Wenhai Liu was born in Hubei, China, in 1969. $\mathrm{He}$ received the B.S. degree in applied physics and electrical engineering from Tsinghua University, China, in 1992 and the M.S. degree in physics from the University of Chicago, IL, in 1996. He is currently pursuing the Ph.D. degree in electrical engineering at the California Institute of Technology, Pasadena.

His research interests are in optical memory and optoelectronic devices.
Jean-Jacques P. Drolet (Associate Member, IEEE) received the B.S. degree from the Universite Laval, P.Q., Canada, in 1990 and the M.S and $\mathrm{Ph} . \mathrm{D}$. degrees in electrical engineering from the California Institute of Technology, Pasadena, in 1992 and 1997, respectively.

His areas of research are in optoelectronic integrated circuits, analog and mixed-signal integrated circuits, and diffractive optical elements. His research thesis at the California Institute of Technology was on silicon/liquid crystal devices. Jean-Jacques is currently with MicroDisplay Corporation, San Pablo, CA.

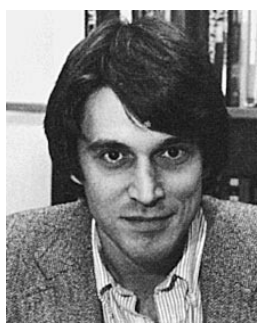

Demetri Psaltis (Senior Member, IEEE) received the B.Sc. degree in electrical engineering and economics in 1974 and the M.Sc. and Ph.D. degrees in electrical engineering in 1975 and 1977, respectively, all from Carnegie-Mellon University, Pittsburgh, PA.

After completing the Ph.D. degree, he remained at Carnegie-Mellon, as a Research Associate and later as a Visiting Assistant Professor, for a period of three years. In 1980, he joined the faculty at the California Institute of Technology, Pasadena, where he is now the Thomas G. Myers Professor of Electrical Engineering. His research interests are in the areas of optical information processing, holography, radar imaging, pattern recognition, neural networks, optical memories, and optical devices. He has authored or coauthored over 320 publications in these areas.

Dr. Psaltis is a Fellow of the Optical Society of America and received the International Commission of Optics Prize in 1989. 